\title{
MicroRNA-330 is downregulated in retinoblastoma and suppresses cell viability and invasion by directly targeting ROCK1
}

\author{
LING WANG, LINA WANG, LIN LI, HONG ZHANG and XUEMAN LYU \\ Department of Ophthalmology, China-Japan Union Hospital of Jilin University, \\ Changchun, Jilin 130033, P.R. China
}

Received June 25, 2018; Accepted February 8, 2019

DOI: $10.3892 / \mathrm{mmr} .2019 .10545$

\begin{abstract}
Abnormal expression of microRNAs (miRNAs /miRs) has been previously reported in various types of human cancer, such as retinoblastoma (RB). Dysregulated miRNAs have been demonstrated to be important epigenetic regulators of numerous biological events associated with RB. Therefore, improved understanding of the precise roles of miRNAs in $\mathrm{RB}$ is required to develop novel therapeutic strategies for the treatment of patients with this disease. In the present study, reverse transcription-quantitative polymerase chain reaction (RT-qPCR) was performed to detect miR-330 expression in RB tissues and cell lines. The effects of miR-330 overexpression on the viability and invasion of RB cells were determined using MTT and Matrigel ${ }^{\circledR}$-based invasion assays, respectively. The mechanisms underlying the activity of miR-330 in RB cells were investigated via bioinformatics analysis, luciferase reporter assays, and RT-qPCR and western blot analyses. It was revealed that the levels of miR-330 expression were significantly downregulated in RB tissues and cell lines compared with in control healthy tissues and cells, respectively. Overexpression of miR-330 in RB cells significantly reduced the viability and invasion of cells in vitro. Additionally, $\rho$-associated coiled-coil containing protein kinase 1 (ROCK1) was identified as a putative target of miR-330 using bioinformatics analysis. Subsequent experiments revealed that miR-330 interacted with the 3'-untranslated region of ROCK1 and downregulated its expression in RB cells. Furthermore, the expression levels of ROCK1 were increased in RB tissues compared with healthy controls and negatively correlated with miR-330 expression. Finally, upregulation of ROCK1 expression reversed the miR-330-induced inhibition of the viability and invasion of
\end{abstract}

Correspondence to: Professor Xueman Lyu, Department of Ophthalmology, China-Japan Union Hospital of Jilin University, 126 Xiantai Road, Changchun, Jilin 130033, P.R. China

E-mail: xm_lyu@yeah.net

Key words: retinoblastoma, microRNA-330, proliferation, invasion, $\rho$-associated coiled-coil containing protein kinase 1
RB cells. Collectively, these results suggested that miR-330 exhibits tumor-suppressor activity in the development of RB by directly targeting ROCK1, indicating that restoration of miR-330 expression may be a promising therapeutic technique in the treatment of patients with RB.

\section{Introduction}

Retinoblastoma (RB) originates from primitive retinal cells and is the most prevalent primary intraocular malignant tumor in children (1). The incidence rate of RB is one case per 15,000-20,000 live births, or $~ 9,000$ novel cases globally every year (2). The symptoms of RB include leukocoria, strabismus, nystagmus, inflamed eyes and loss of sight, and the clinical presentation depends almost entirely on the location of the tumor (3). Marked improvements in surgical resection and chemotherapy have been made, and remain as the standard treatment options; however, the clinical outcome for patients with RB remains unsatisfactory due to the spread of tumors to the brain via the optic nerve $(4,5)$. The RB1 gene has been identified as defective in the pathogenesis of RB (6); however, the complex mechanisms underlying the formation and progression of RB remain unclear. Therefore, improved understanding of the mechanisms underlying the pathogenesis and development of RB is urgently required for the identification of novel therapeutic strategies for the treatment of patients with this disease.

MicroRNAs (miRNAs/miRs) are a series of single-stranded noncoding RNA molecules comprising 19-25 nucleotides (7). A total of 1,881 precursor and 2,588 mature miRNAs have been identified in the human genome, according to miRBase (http://www.mirbase.org/index.shtml) (8). Mature miRNAs act as important modulators of gene expression by directly interacting with complementary sequences in the 3 -untranslated regions (3'-UTRs) of their target genes, suppressing translation and inducing the degradation of mRNAs (9). It has been reported that miRNAs are aberrantly expressed in almost all types of human cancer, and their dysregulation contributes to tumorigenesis and development (10-12). In particular, a large number of miRNAs have been identified as dysregulated in $\mathrm{RB}$; these miRNAs are involved in the regulation of a wide range of biological behaviors, including the proliferation, cell 
cycle, apoptosis, invasion, metastasis and chemoresistance of tumor cells (13-15). Therefore, investigation into the expression profiles and roles of miRNAs in RB may aid the identification of novel targets in the diagnosis or treatment of RB.

Numerous studies have reported that the dysregulated expression of miR-330 is involved in the onset and progression of various human cancers, including breast $(16,17)$, esophageal (18) and lung cancer $(19,20)$, and glioblastoma (21); however, the expression profile, roles and underlying mechanisms of miR-330 in RB remain unclear. Therefore, the present study aimed to investigate the expression of miR-330 in $\mathrm{RB}$ tissues and cell lines, determine the roles served by miR-330 in $\mathrm{RB}$ cells and reveal the underlying mechanisms.

\section{Materials and methods}

Clinical specimens. The present study was approved by the Ethics Committee of the China-Japan Union Hospital of Jilin University (Changchun, China). Written informed consent was obtained from all patients that participated in the study. RB tissues were collected from 24 patients with RB (16 males and 8 females; aged 14-35 years) that had not been treated with chemotherapy and radiotherapy prior to surgical treatment at the China-Japan Union Hospital of Jilin University. Normal retinal tissues were obtained from 7 patients $(5$ males and 2 females; aged 26-57 years) who were diagnosed with globe rupture and treated via enucleation. Tissues were collected between May 2015 and October 2017. All tissues were flash frozen in liquid nitrogen and stored at $-80^{\circ} \mathrm{C}$ until further use.

Cell lines. The human RB cell lines (Y79, SO-RB50 and Weri-RB1) and normal retinal pigmented epithelium cell line ARPE-19 were all obtained from the American Type Culture Collection (Manassas, VA, USA). The cells were cultured in Dulbecco's Modified Eagle's medium (DMEM) containing $10 \% \mathrm{v} / \mathrm{v}$ heat-inactivated fetal bovine serum (FBS) and $1 \% \mathrm{v} / \mathrm{v}$ penicillin-streptomycin mixture (all from Gibco; Thermo Fisher Scientific, Inc., Waltham, MA, USA). The cell lines were cultured at $37^{\circ} \mathrm{C}$ in a humidified incubator supplemented with $5 \% \mathrm{CO}_{2}$.

Cell transfection. The miR-330 mimics and miRNA mimics negative control (miR-NC) used in the study were purchased from Shanghai GenePharma Co., Ltd. (Shanghai, China). The miR-330 mimics sequence was 5'-GCAAAGCACACGGCC UGCAGAGA-3', and the miR-NC sequence was 5'-UUCUCC GAACGUGUCACGUTT-3'. The $\rho$-associated coiled-coil containing protein kinase 1 (ROCK1) overexpression plasmid pCMV-ROCK1 and blank plasmid pCMV were designed and chemically synthesized by Obio Technology Corp., Ltd. (Shanghai, China). Y79 and Weri-RB1 cells $\left(5 \times 10^{5}\right.$ cells/well) were seeded into 6-well plates and incubated overnight at $37^{\circ} \mathrm{C}$ under $5 \% \mathrm{CO}_{2}$. Y79 and Weri-RB1 cells were transfected with miR-330 mimics (100 pmol), miR-NC (100 pmol), pCMV-ROCK1 $(4 \mu \mathrm{g})$ or pCMV $(4 \mu \mathrm{g})$ using Lipofectamine ${ }^{\circledR}$ 2000 (Invitrogen; Thermo Fisher Scientific, Inc.) according to the manufacturer's protocols. At $6 \mathrm{~h}$ following transfection, cells were washed with PBS (Gibco; Thermo Fisher Scientific, Inc.), and DMEM with $10 \%$ FBS was added to each well. Reverse transcription-quantitative polymerase chain reaction (RT-qPCR) analysis and MTT assays were conducted following incubation at $37^{\circ} \mathrm{C}$ for $24 \mathrm{~h}$. Matrigel ${ }^{\circledR}$-based invasion assays and western blot analysis were conducted at 48 and $72 \mathrm{~h}$ post-transfection, respectively.

$R T$-qPCR analysis. Total RNA was isolated from tissue samples and cultured cells using TRIzol ${ }^{\circledR}$ reagent (Thermo Fisher Scientific, Inc.) according to the manufacturer's protocols. To determine the expression levels of miRNA, cDNA was produced from total RNA using a miScript Reverse Transcription kit (Qiagen GmbH, Hilden, Germany), according to the manufacturer's protocols. Subsequently, qPCR was performed to determine miR-330 expression using a miScript SYBR ${ }^{\circledR}$ Green PCR kit (Qiagen GmbH). The thermocycling conditions for qPCR were as follows: $95^{\circ} \mathrm{C}$ for $2 \mathrm{~min}, 95^{\circ} \mathrm{C}$ for $10 \mathrm{sec}, 55^{\circ} \mathrm{C}$ for $30 \mathrm{sec}$ and $72^{\circ} \mathrm{C}$ for $30 \mathrm{sec}$, for 40 cycles. For the determination of ROCK1 mRNA expression, total RNA was reverse-transcribed into cDNA using an M-MLV Reverse Transcription system (Promega Corporation, Madison, WI, USA) according to the manufacturer's protocols, which was then subjected to qPCR using an SYBR Green I mix (Takara Biotechnology Co., Ltd., Dalian, China). The thermocycling conditions for qPCR were as follows: $95^{\circ} \mathrm{C}$ for $10 \mathrm{~min}$, followed by 40 cycles of $95^{\circ} \mathrm{C}$ for $15 \mathrm{sec}$ and $60^{\circ} \mathrm{C}$ for $1 \mathrm{~min}$. The levels of miR-330 and ROCK1 mRNA were analyzed using the $2^{-\Delta \Delta C q}$ method (22), and normalized to the expression levels of U6 small nuclear RNA and GAPDH, respectively. The primers were designed as follows: miR-330, forward 5'-GGGCTCGAGCCA CTCACCCACACTGAAGA-3', reverse, 5'-GGGGCGGCC GCGTTTCTCCCTCTGCTTGACG-3'; U6, forward 5'-GCT TCGGCAGCACATATACTAAAAT-3', reverse, 5'-CGCTTC ACGAATTTGCGTGTCAT-3'; ROCK1, forward 5'-AGGAAG GCGGACATATTGATCCCT-3', reverse, 5'-AGACGATAG TTGGGTCCCGGC-3'; and GAPDH, forward 5'-CAGCCT CAAGATCATCAGCA-3' and reverse, 5'-GTCTTCTGGGTG GCAGTGAT-3'. RT-qPCR experiments were performed in triplicate and repeated at least three times.

MTT assay. Transfected Y79 and Weri-RB1 cells were collected following $24 \mathrm{~h}$ of incubation and plated in a 96-well plate at a density of $3 \times 10^{3}$ cells/well. Y79 and Weri-RB1 cells were then incubated at $37^{\circ} \mathrm{C}$ with $5 \% \mathrm{CO}_{2}$. MTT assays were performed to determine the viability of cells at $0,24,48$ and $72 \mathrm{~h}$ following inoculation at $37^{\circ} \mathrm{C}$. In detail, $20 \mu \mathrm{l}$ of MTT solution ( $5 \mathrm{mg} / \mathrm{ml}$; Sigma-Aldrich; Merck KGaA, Darmstadt, Germany) was added to each well, prior to incubation at $37^{\circ} \mathrm{C}$ for $4 \mathrm{~h}$. The culture medium was then discarded, and $150 \mu \mathrm{l}$ dimethyl sulfoxide (Beyotime Institute of Biotechnology, Shanghai, China) was added to dissolve the formazan crystals. A microplate reader (Bio-Rad Laboratories, Inc., Hercules, CA, USA) was used to detect the absorbance at $490 \mathrm{~nm}$. MTT assays were performed in triplicate and repeated at least three times.

Matrigel-based invasion assay. The invasive ability of cells was determined using 24-well Transwell inserts (8- $\mu \mathrm{m}$ pores) coated with Matrigel (BD Biosciences, Franklin Lakes, NJ, USA). Transfected Y79 and Weri-RB1 cells were harvested following $48 \mathrm{~h}$ of incubation and re-suspended in FBS-free DMEM. A total of $1 \times 10^{5}$ cells were plated in the upper chamber 
A

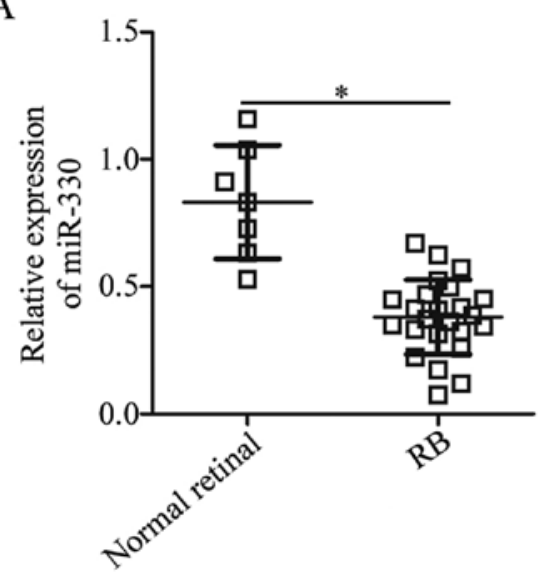

B

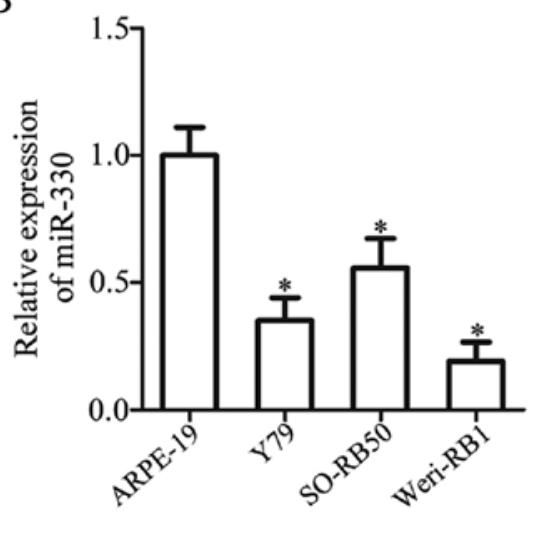

Figure 1. miR-330 is downregulated in RB tissues and cell lines. (A) RT-qPCR was used to analyze miR-330 expression in 24 RB and 7 normal retinal tissues. ${ }^{*} \mathrm{P}<0.05$. (B) Expression levels of miR-330 were determined in three human RB cell lines (Y79, SO-RB50 and Weri-RB1) and one normal retinal pigmented epithelium cell line (ARPE-19) via RT-qPCR. Data are presented as the mean \pm standard deviation of at least three experiments. ${ }^{*}<0.05$ vs. ARPE-19. miR-330, microRNA-330; RB, retinoblastoma; RT-qPCR, reverse transcription-quantitative polymerase chain reaction.

of Transwell inserts, whereas DMEM with 20\% FBS (500 $\mu \mathrm{l}$; Gibco; Thermo Fisher Scientific, Inc.) was added to the lower chambers. Non-invasive cells were gently removed $24 \mathrm{~h}$ later with a cotton swab. Invasive cells were fixed with $4 \%$ paraformaldehyde at room temperature for $20 \mathrm{~min}$, and stained with $0.05 \%$ crystal violet (Beyotime Institute of Biotechnology) at room temperature for $20 \mathrm{~min}$. The average number of invasive cells in five randomly selected fields was calculated for each insert under a light microscope (magnification, x200; Olympus Corporation, Tokyo, Japan). Each experiment was repeated at least three times.

Bioinformaticsanalysisandluciferasereporterassay.miRanda (version 3.3a; http://www.microrna.org/microrna/home.do) and TargetScan (Release 6.0; www.targetscan.org) were employed to identify putative targets of miR-330. It was revealed that miR-330 may directly bind to the sequence at 141-147 bp in the 3'-UTR of ROCK1. Wild-type and mutant 3'-UTR fragments of ROCK1 were generated by Shanghai GenePharma Co., Ltd. and subcloned into a pmirGLO dual-luciferase reporter vector (Promega Corporation). Y79 and Weri-RB1 cells were inoculated in 24-well plates 1 night prior to transfection. Luciferase plasmids containing the wild-type or mutant 3'-UTR of ROCK1 were cotransfected with miR-330 mimics or miR-NC into cells using Lipofectamine 2000, according to the manufacturer's protocols. Following culture for $48 \mathrm{~h}$ at $37^{\circ} \mathrm{C}$, the Dual-Luciferase Reporter Assay System (Promega Corporation) was used to evaluate luciferase activity. The relative luciferase activity was normalized to Renilla luciferase activity. Each experiment was repeated at least three times.

Western blot analysis. Total protein was extracted from tissues or cells using radioimmunoprecipitation assay lysis buffer (Beyotime Institute of Biotechnology). The concentration of total protein was determined using a Bicinchoninic Acid Protein Quantification kit (Vazyme, Piscataway, NJ, USA). Proteins $(20 \mu \mathrm{g} /$ lane) were separated via $10 \%$ SDS-PAGE and transferred to polyvinylidene difluoride membranes (Beyotime Institute of Biotechnology). Then, the membranes were blocked at room temperature for $2 \mathrm{~h}$ in Tris-buffered saline containing $0.1 \%$ Tween-20 (TBST) with 5\% dried skimmed milk, followed by incubation with primary antibodies overnight at $4^{\circ} \mathrm{C}$. The following primary antibodies from Abcam (Cambridge, UK) were used: Rabbit anti-human ROCK1 (1:1,000; cat. no. ab45171) and rabbit anti-human GAPDH (1:1,000; cat. no. ab181603). Following three washes with TBST, the membranes were further incubated with a goat anti-rabbit horseradish peroxidase-conjugated secondary antibody (1:5,000; ab6721, Abcam) at room temperature for $2 \mathrm{~h}$. Protein bands were visualized using a Pierce ${ }^{\mathrm{TM}}$ Fast Western Blot kit (Pierce; Thermo Fisher Scientific, Inc.). GAPDH was used as a loading control. All experiments were repeated at least three times. Quantity One version 4.62 software (Bio-Rad Laboratories, Inc.) was used to quantify protein expression.

Statistical analysis. Statistical analysis was conducted using SPSS v.16.0 (SPSS, Inc., Chicago, IL, USA). All experimental data were presented as the mean \pm standard deviation from at least three independent experiments. Differences between groups were analyzed using Student's t-tests or one-way analyses of variance followed by a Tukey's post hoc test. Pearson correlation analysis was performed to investigate the association between miR-330 and ROCK1 expression in RB tissues. $\mathrm{P}<0.05$ was considered to indicate a statistically significant difference.

\section{Results}

miR-330 is downregulated in RB tissues and cell lines. The expression of miR-330 in $24 \mathrm{RB}$ and 7 normal retinal tissues was determined. RT-qPCR analysis revealed that the levels of miR-330 expression was significantly downregulated in $\mathrm{RB}$ tissues compared with in normal retinal tissues $(\mathrm{P}<0.05$; Fig. 1A). Additionally, the expression levels of miR-330 in three human RB cell lines (Y79, SO-RB50 and Weri-RB1) were significantly decreased compared with in ARPE-19 cells $(\mathrm{P}<0.05$; Fig. 1B). The results suggested that the downregulation of miR-330 is an important event in RB. 
A

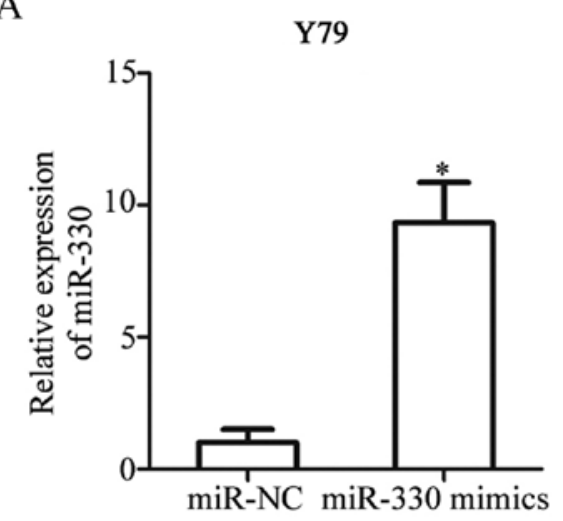

B

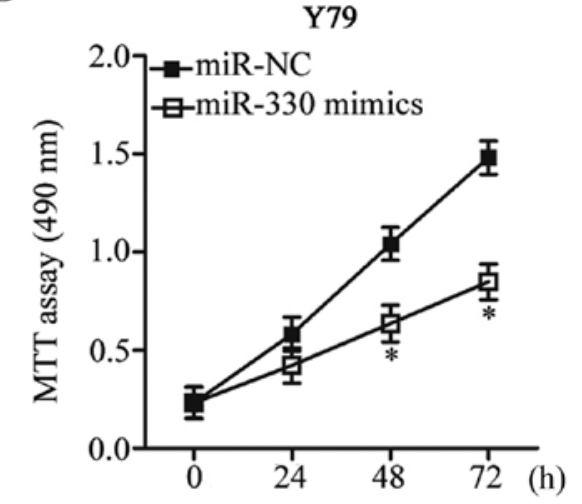

$\mathrm{C}$
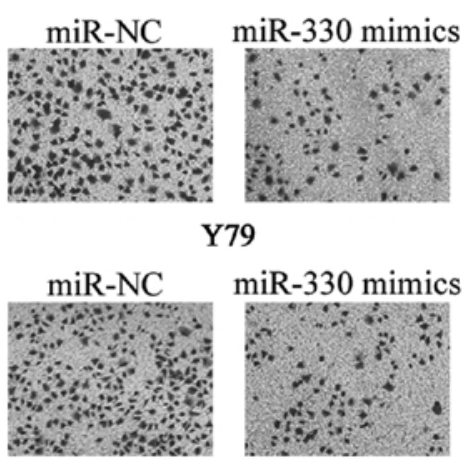

Weri-RB1
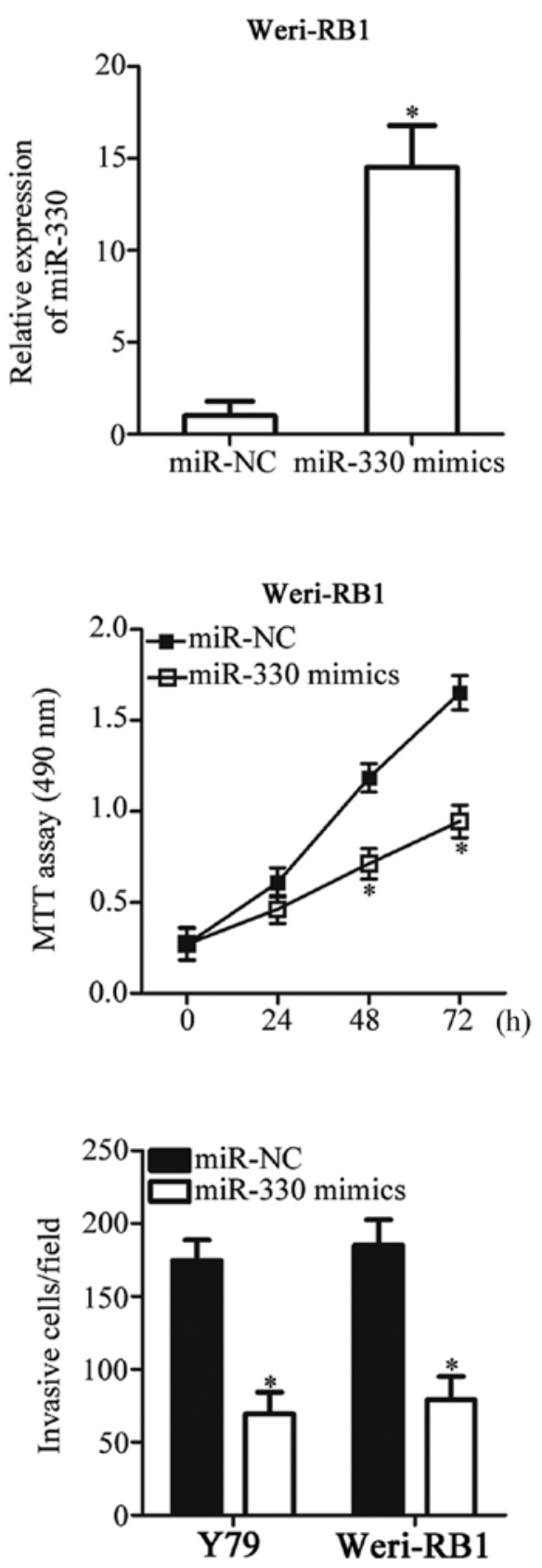

Figure 2. Inhibitory effects of miR-330 overexpression on the viability and invasive ability of retinoblastoma cells. (A) Y79 and Weri-RB1 cells were treated with miR-330 mimics or miR-NC. Reverse transcription-quantitative polymerase chain reaction was conducted to determine miR-330 expression at $48 \mathrm{~h}$ post-transfection. (B) MTT assays were performed to determine the viability of Y79 and Weri-RB1 cells transfected with miR-330 mimics or miR-NC. (C) Invasion of miR-330 mimics or miR-NC-transfected Y79 and Weri-RB1 cells as determine using Matrigel assays. Data are presented as the mean \pm standard deviation of at least three independent experiments. ${ }^{*} \mathrm{P}<0.05$ vs. miR-NC. miR, microRNA; NC, negative control.

miR-330 overexpression inhibits $R B$ cell viability and invasion in vitro. To investigate the potential roles of miR-330 in RB, Y79 and Weri-RB1 cells, due to their relatively reduced miR-330 expression compared with SO-RB50 cells, were selected for subsequent experiments and transfected with miR-330 mimics or miR-NC. miR-330 was effectively overexpressed in miR-330 mimics-transfected Y79 and Weri-RB1 cells $(\mathrm{P}<0.05$; Fig. 2A). MTT assays were subsequently performed to determine the effects of upregulation of miR-330 on the viability of RB cells. It was revealed that transfection with miR-330 mimics significantly reduced the viability of Y79 and Weri-RB1 cells compared with miR-NC
( $\mathrm{P}<0.05$; Fig. 2B). Furthermore, the invasive abilities of Y79 and Weri-RB1 cells transfected with miR-330 mimics or miR-NC were further determined using Matrigel invasion assays. Overexpression of miR-330 significantly suppressed the invasion of Y79 and Weri-RB1 cells compared with cells transfected with miR-NC $(\mathrm{P}<0.05$; Fig. $2 \mathrm{C})$. The results indicated that miR-330 serves a tumor suppressor role in the progression of RB.

miR-330 directly targets ROCK1 and decreases its expression in $R B$ cells. Bioinformatics analysis was employed to identify the putative targets of miR-330. Analysis indicated 
A

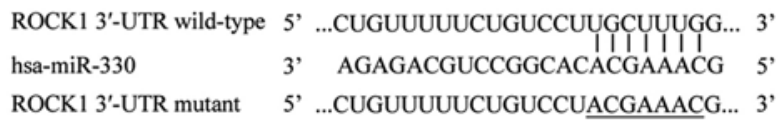

B



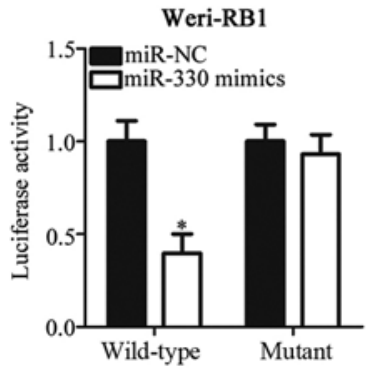

C

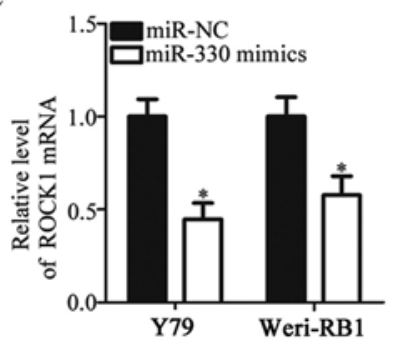

D

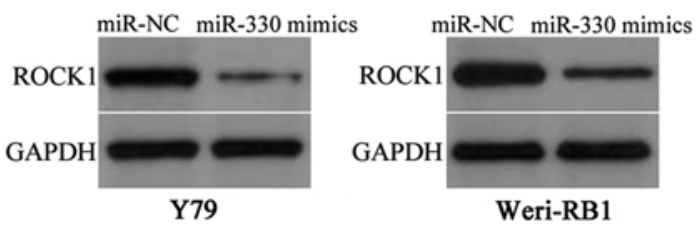

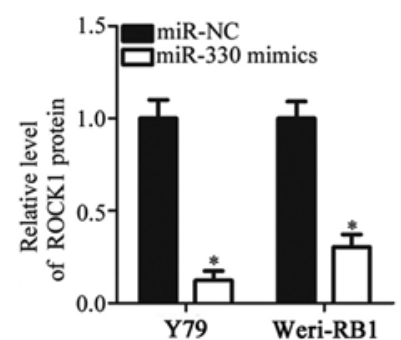

Figure 3. ROCK1 is a direct target gene of miR-330 in retinoblastoma cells. (A) Putative wild-type and mutant miR-330 binding sites in the 3'-UTR of ROCK1. (B) Y79 and Weri-RB1 cells were co-transfected with luciferase plasmids containing the wild-type or mutant 3'-UTR of ROCK1 and miR-330 mimics or miR-NC. Luciferase activity was measured by a dual-luciferase reporter assay after $48 \mathrm{~h}$ of incubation. Expression levels of ROCK1 (C) mRNA and (D) protein in Y79 and Weri-RB1 cells transfected with miR-330 mimics or miR-NC. Data are presented as the mean \pm standard deviation of at least three independent experiments. ${ }^{*} \mathrm{P}<0.05$ vs. miR-NC. miR, microRNA; NC, negative control; ROCK1, $\rho$-associated coiled-coil containing protein kinase 1 ; UTR, untranslated region.

A

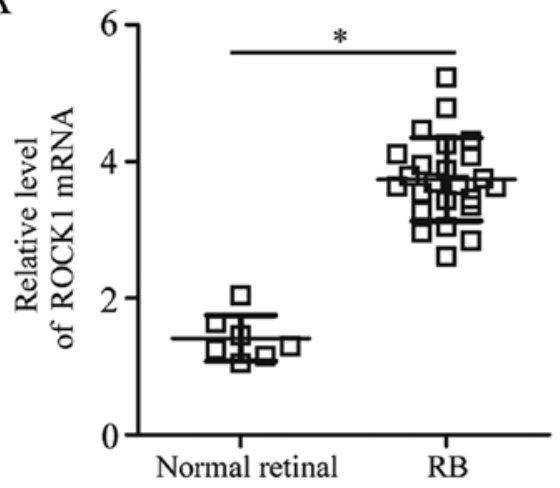

B

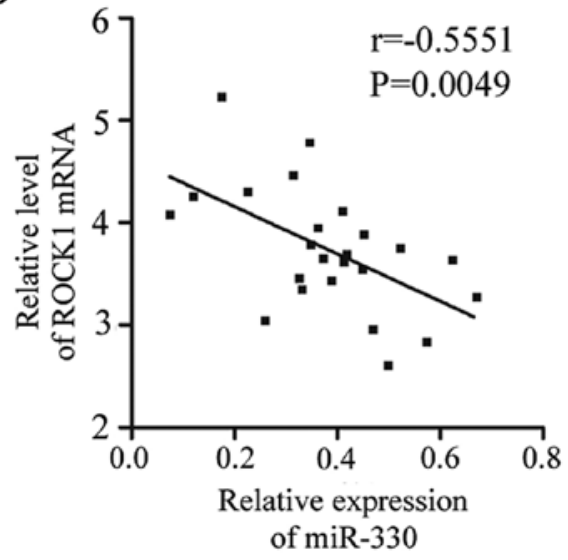

Figure 4. ROCK1 is upregulated in RB tissues and is negatively correlated with miR-330 expression. (A) Reverse transcription-quantitative polymerase chain reaction analysis was conducted to determine the levels of ROCK1 mRNA expression in 24 RB and 7 normal retinal tissues. Data are presented as the mean \pm standard deviation. ${ }^{*} \mathrm{P}<0.05$. (B) Pearson correlation analysis of the association between miR-330 and ROCK1 mRNA levels in RB tissues. miR-330, microRNA-330; RB, retinoblastoma; ROCK1, $\rho$-associated coiled-coil containing protein kinase 1.

that there was a binding site for miR-330 within the 3'-UTR region of ROCK1 (Fig. 3A). ROCK1 was selected for further experimentation as previous studies have reported ROCK1 to be associated with the genesis and development of $\operatorname{RB}(23,24)$. A luciferase reporter assay was conducted to verify whether miR-330 was able to bind to the 3'-UTR region of ROCK1. The results revealed that overexpression of miR-330 significantly decreased the luciferase activity of plasmids containing the wild-type, but not mutant 3'-UTR of ROCK1 in Y79 and Weri-RB1 cells compared with cells transfected with miR-NC $(\mathrm{P}<0.05$; Fig. 3B). To investigate whether miR-330 regulates the expression of ROCK1 in RB cells, RT-qPCR and western blot analysis were performed in Y79 and Weri-RB1 cells following transfection with miR-330 mimics or miR-NC. It was revealed that miR-330 mimics-transfected Y79 and Weri-RB1 cells exhibited significantly decreased ROCK1 mRNA $(\mathrm{P}<0.05$; Fig. 3C) and protein $(\mathrm{P}<0.05$; Fig. 3D) expression compared with cells transfected with miR-NC. Collectively, these results indicated that ROCK1 is a target gene of miR-330 in RB cells.

Expression of miR-330 is inversely correlated with ROCK1 expression in $R B$ tissues. To further investigate the association between miR-330 and ROCK1 in RB, RT-qPCR analysis was performed using $\mathrm{RB}$ and normal retinal tissues. ROCK1 
A

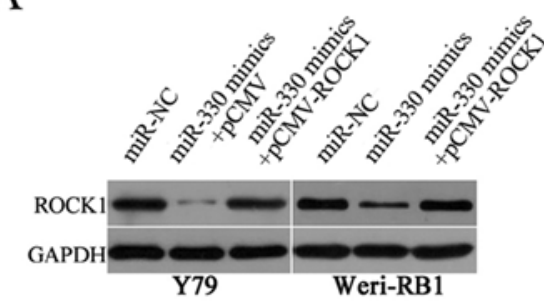



$\mathrm{B}$
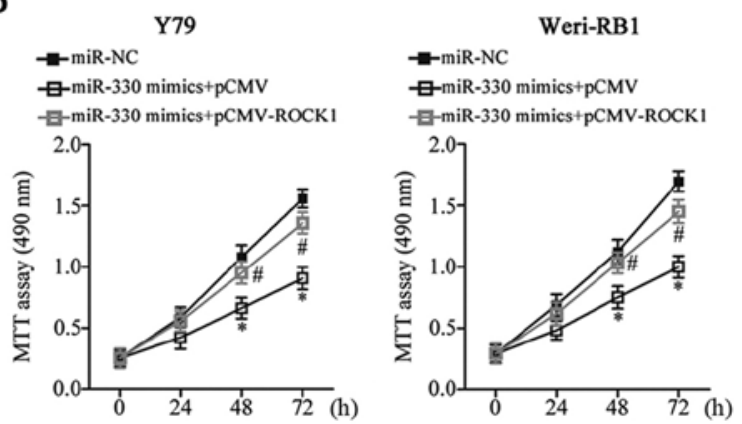

C
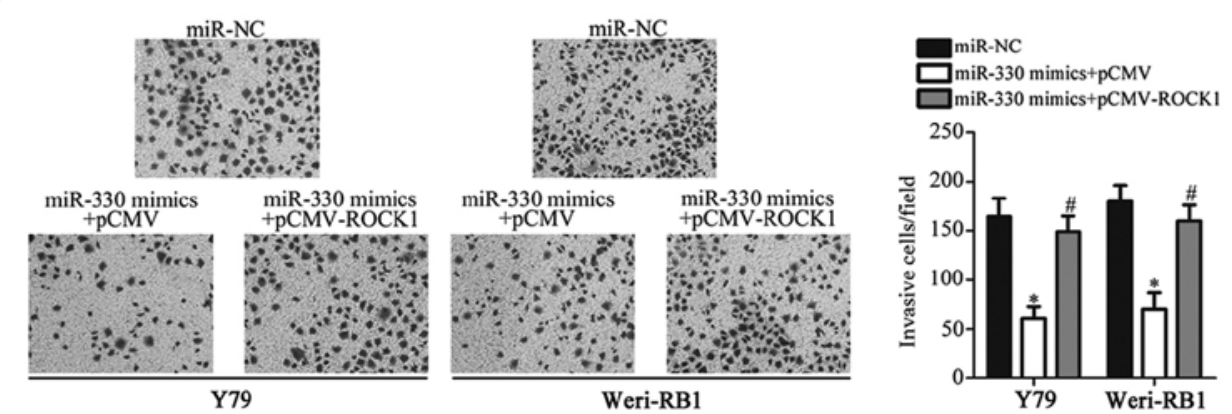

Figure 5. ROCK1 rescue reverses the suppressive effects of miR-330 overexpression on retinoblastoma cells. Y79 and Weri-RB1 cells were co-transfected with miR-330 mimics and pCMV or pCMV-ROCK1. (A) ROCK1 protein expression $72 \mathrm{~h}$ following transfection, as determined by western blotting. (B) MTT and (C) Matrigel invasion assays were conducted to investigate the viability and invasive abilities of transfected cells, respectively. Data are presented as the mean \pm standard deviation of at least three independent experiments. ${ }^{*} \mathrm{P}<0.05$ vs. miR-NC. ${ }^{~} \mathrm{P}<0.05$ vs. miR-330 mimics+pCMV. miR, microRNA; NC, negative control; ROCK1, $\rho$-associated coiled-coil containing protein kinase 1.

mRNA expression levels were upregulated in RB tissues compared with in normal retinal tissues $(\mathrm{P}<0.05$; Fig. 4A). Additionally, Pearson correlation analysis revealed an inverse correlation between the levels of miR-330 and ROCK1 mRNA expression in RB tissues ( $r=-0.5551, \mathrm{P}=0.0049$; Fig. 4B). The results indicated that the upregulation of ROCK1 in RB tissues is associated with the downregulation of miR-330.

Rescue of ROCK1 expression reverses the suppressive effects of miR-330 overexpression in $R B$ cells. Rescue experiments were conducted to determine whether ROCK1 is associated with the tumor-suppressive roles of miR-330 in RB cells. Y79 and Weri-RB1 cells were transfected with miR-330 mimics in combination with pCMV or pCMV-ROCK1. The downregulation of ROCK1 expression in Y79 and Weri-RB1 cells induced by miR-330 overexpression was rescued by co-transfection with pCMV-ROCK1 ( $\mathrm{P}<0.05$; Fig. 5A). Additionally, MTT and Matrigel invasion assays revealed that rescue of ROCK1 expression eliminated the inhibitory effects of miR-330 overexpression on the viability $(\mathrm{P}<0.05$; Fig. $5 \mathrm{~B})$ and invasion $(\mathrm{P}<0.05$; Fig. 5C) of Y79 and Weri-RB1 cells. The results indicated that miR-330 serves tumor-suppressor roles in RB cells by downregulating ROCK1 expression.

\section{Discussion}

The abnormal expression of miRNAs has been reported in almost all types of human cancer, including RB (25-27). Dysregulated miRNAs have been reported to be important epigenetic regulators of numerous biological events associated with RB, acting as oncogenes or tumor suppressors (13). Therefore, determining the detailed roles of miRNAs in RB could aid the identification of potential therapeutic approaches in the treatment of patients with RB. miR-330 was reported to be upregulated in breast cancer, and increased miR-330 expression was significantly associated with tumor, node and metastasis stage, and lymph node metastasis (16). Patients with breast cancer and high levels of miR-330 expression demonstrated reduced 5-year overall survival than patients with low miR-330 expression levels (16). Additionally, univariate and multivariate regression analyses have identified the expression of miR-330 as an independent prognostic factor for patients with breast cancer $(16,17)$. miR-330 has also been reported to be upregulated in esophageal cancer (18), non-small cell lung cancer (NSCLC) $(19,20)$ and glioblastoma (21). Conversely, miR-330 expression is downregulated in prostate cancer (28), osteosarcoma (29) and colorectal cancer (30); however, its expression status in RB remains unclear. These contradictory observations indicate that miR-330 exhibits tissue-specific expression profiles in human malignant tumors. In the present study, it was revealed that miR-330 expression was downregulated in RB tissues and cell lines, suggesting that miR-330 may be a novel biomarker for the diagnosis of this specific type of cancer.

miR-330 has been reported to serve oncogenic roles in the process of carcinogenesis and cancer progression. For example, miR-330 overexpression promoted the metastasis of breast cancer in vitro (17). In esophageal cancer, upregulation 
of miR-330 increased the proliferation, survival and metastasis of cells in vitro, and promoted tumor growth in vivo (18). Additionally, in NSCLC, overexpression of miR-330 enhanced tumor growth and metastasis in vitro and in vivo $(19,20)$, whereas in glioblastoma, miR-330 overexpression promoted the proliferation and motility of cells, induced cell cycle arrest and suppressed the apoptosis of cells in vitro (21). Conversely, miR-330 served as a tumor suppressor in osteosarcoma (29), and prostate (28) and colorectal cancer (30), demonstrating the tissue-specific activity of miR-330. In the present study, MTT and Matrigel invasion assays were employed to determine the regulatory roles of miR-330 in RB cells; it was revealed that miR-330 acts as a tumor suppressor in RB cells by inhibiting the viability and invasion of cells in vitro. These findings suggested that miR-330 may be a therapeutic target in the treatment of patients with $\mathrm{RB}$.

Numerous targets of miR-330 have been identified, including collagen and calcium-binding epidermal growth factor domain-containing protein 1 (17) and programmed cell death protein 4 (18) in breast cancer, early growth response protein 2 (19) and glutamate ionotropic receptor AMPA type subunit 3 (20) in lung cancer, SH3 domain containing GRB2 like 2, endophilin-A1 (21) in glioblastoma, specificity protein 1 (28) in prostate cancer, B lymphoma Mo-MLV insertion region homolog 1 (29) in osteosarcoma and thymidylate synthetase (30) in colorectal cancer. In the present study, ROCK1 was validated as a direct target gene of miR-330 in RB cells. TargetScan and miRanda were employed to identify putative targets of miR-330. It was considered that miR-330 may directly bind to the 141-147 bp sequence in the 3'-UTR of ROCK1. Then, luciferase reporter assays, RT-qPCR and western blotting demonstrated that miR-330 could directly target the 3'-UTR of ROCK1 and decrease its expression in RB cells. Furthermore, it was revealed that ROCK1 was upregulated in RB tissues and its expression was inversely correlated with miR-330 expression. Finally, restoration of ROCK1 expression eliminated the suppressive effects of miR-330 overexpression on the malignant phenotypes of RB cells.

ROCK1, located on 18q11.1, is an essential downstream effector of the Rho small guanosine 5'-triphosphatase and serves as a molecular switch, binding GTP and guanosine 5'-diphosphate to regulate various biological behaviors (31-33). It is frequently overexpressed in a variety of human cancers, including pancreatic cancer (34), nasopharyngeal carcinoma (35), bladder cancer (36) and osteosarcoma (37). Additionally, the present study reported that ROCK1 was demonstrated to be upregulated in RB. The dysregulation of ROCK1 is involved in the initiation and progression of RB, and regulates a number of physiological and pathological processes, including the proliferation, invasion, adhesion and apoptosis of cells $(23,24)$. In the present study, it was demonstrated that miR-330 targets ROCK1 to inhibit the progression of RB cells; thus, the miR-330/ROCK1 axis may be a potential therapeutic target in the treatment of patients with RB.

In conclusion, the findings from the present study indicated that miR-330 may function as a tumor suppressor in RB by directly targeting ROCK1 to inhibit the viability and invasion of cells. Further investigation into the mechanisms underlying the precise functions of miR-330 in RB may aid the identification of therapeutic approaches for patients with this malignancy. A single miRNA can regulate numerous targets, and a single gene can be negatively modulated by various miRNAs (9); however, in the present study, only ROCK1 was identified as a direct target gene of miR-330 in RB cells. Further studies are required to address this issue. Furthermore, the patient sample size was small. Therefore, analysis of miR-330/ROCK expression in additional tissues from patients with RB is required to further validate these findings.

\section{Acknowledgements}

Not applicable.

\section{Funding}

Not funding was received.

\section{Availability of data and materials}

The datasets used and/or analyzed during the current study are available from the corresponding author upon reasonable request.

\section{Authors' contributions}

LingW and XL designed the present study. LingW and LinaW conducted RT-qPCR and western blot analyses, and the luciferase reporter assays. LL and HZ performed the MTT and Matrigel-based invasion assays. All authors have read and approved the final draft.

\section{Ethics approval and consent to participate}

The present study was approved by the Ethics Committee of China-Japan Union Hospital of Jilin University (Changchun, China), and was performed in accordance with the Declaration of Helsinki and the guidelines of the Ethics Committee of China-Japan Union Hospital of Jilin University. Written informed consent was obtained from all patients for the use of their clinical tissues.

\section{Patient consent for publication}

Not applicable.

\section{Competing interests}

The authors declare that they have no competing interests.

\section{References}

1. Jabbour P, Chalouhi N, Tjoumakaris S, Gonzalez LF, Dumont AS, Chitale R, Rosenwasser R, Bianciotto CG and Shields C: Pearls and pitfalls of intraarterial chemotherapy for retinoblastoma. J Neurosurg Pediatr 10: 175-181, 2012.

2. Kivelä T: The epidemiological challenge of the most frequent eye cancer: Retinoblastoma, an issue of birth and death. $\mathrm{Br} \mathrm{J}$ Ophthalmol 93: 1129-1131, 2009.

3. Abramson DH, Beaverson K, Sangani P, Vora RA, Lee TC, Hochberg HM, Kirszrot J and Ranjithan M: Screening for retinoblastoma: Presenting signs as prognosticators of patient and ocular survival. Pediatrics 112: 1248-1255, 2003. 
4. Meel R, Radhakrishnan V and Bakhshi S: Current therapy and recent advances in the management of retinoblastoma. Indian $\mathrm{J}$ Med Paediatr Oncol 33: 80-88, 2012.

5. Abramson DH, Marr BP, Brodie S, Dunkel IJ and Gobin PY: Intraarterial chemotherapy for kissing macula tumors in retinoblastoma. Retin Cases Brief Rep 6: 209-211, 2012.

6. Benavente CA and Dyer MA: Genetics and epigenetics of human retinoblastoma. Annu Rev Pathol 10: 547-562, 2015

7. Munker R and Calin GA: MicroRNA profiling in cancer. Clin Sci (Lond) 121: 141-158, 2011.

8. Zheng Y, Lu X, Xu L, Chen Z, Li Q and Yuan J: MicroRNA-675 promotes glioma cell proliferation and motility by negatively regulating retinoblastoma 1. Hum Pathol 69: 63-71, 2017.

9. Bagnyukova TV, Pogribny IP and Chekhun VF: MicroRNAs in normal and cancer cells: A new class of gene expression regulators. Exp Oncol 28: 263-269, 2006.

10. Laengsri V, Kerdpin U, Plabplueng C, Treeratanapiboon L and Nuchnoi P: Cervical cancer markers: Epigenetics and microRNAs. Lab Med 49: 97-111, 2018.

11. Lou W, Liu J, Gao Y, Zhong G, Chen D, Shen J, Bao C, Xu L, Pan J, Cheng J, et al: MicroRNAs in cancer metastasis and angiogenesis. Oncotarget 8: 115787-115802, 2017.

12. Ramassone A, Pagotto S, Veronese A and Visone R: Epigenetics and MicroRNAs in cancer. Int J Mol Sci 19: pii: E459, 2018.

13. Golabchi K, Soleimani-Jelodar R, Aghadoost N, Momeni F Moridikia A, Nahand JS, Masoudifar A, Razmjoo H and Mirzaei H: MicroRNAs in retinoblastoma: Potential diagnostic and therapeutic biomarkers. J Cell Physiol 233: 3016-3023, 2018.

14. Singh U, Malik MA, Goswami S, Shukla S and Kaur J: Epigenetic regulation of human retinoblastoma. Tumour Biol 37 14427-14441, 2016.

15. Huang J, Yang Y, Fang F and Liu K: MALAT1 modulates the autophagy of retinoblastoma cell through miR-124-mediated stx17 regulation. J Cell Biochem 119: 3853-3863, 2018.

16. Wang H, Chen SH, Kong P, Zhang LY, Zhang LL, Zhang NQ and $\mathrm{Gu} \mathrm{H}$ : Increased expression of miR-330-3p: A novel independent indicator of poor prognosis in human breast cancer. Eur Rev Med Pharmacol Sci 22: 1726-1730, 2018.

17. Mesci A, Huang X, Taeb S, Jahangiri S, Kim Y, Fokas E, Bruce J, Leong HS and Liu SK: Targeting of CCBE1 by miR-330-3p in human breast cancer promotes metastasis. Br J Cancer 116 1350-1357, 2017

18. Meng H, Wang K, Chen X, Guan X, Hu L, Xiong G, Li J and Bai Y: MicroRNA-330-3p functions as an oncogene in human esophageal cancer by targeting programmed cell death 4 . Am J Cancer Res 5: 1062-1075, 2015.

19. Liu X, Shi H, Liu B, Li J, Liu Y and Yu B: miR-330-3p controls cell proliferation by targeting early growth response 2 in non-small-cell lung cancer. Acta Biochim Biophys Sin (Shanghai) 47: 431-440, 2015.

20. Wei CH, Wu G, Cai Q, Gao XC, Tong F, Zhou R, Zhang RG, Dong JH, Hu Y and Dong XR: MicroRNA-330-3p promotes cell invasion and metastasis in non-small cell lung cancer through GRIA3 by activating MAPK/ERK signaling pathway. J Hematol Oncol 10: 125, 2017.

21. Qu S, Yao Y, Shang C, Xue Y, Ma J, Li Z and Liu Y: MicroRNA-330 is an oncogenic factor in glioblastoma cells by regulating SH3GL2 gene. PLoS One 7: e46010, 2012.
22. Livak KJ and Schmittgen TD: Analysis of relative gene expression data using real-time quantitative PCR and the 2(-Delta Delta C(T)) method. Methods 25: 402-408, 2001.

23. Wang J, Liu XH, Yang ZJ, Xie B and Zhong YS: The effect of ROCK-1 activity change on the adhesive and invasive ability of Y79 retinoblastoma cells. BMC Cancer 14: 89, 2014.

24. Wu S, Ai N, Liu Q and Zhang J: MicroRNA-448 inhibits the progression of retinoblastoma by directly targeting ROCK1 and regulating PI3K/AKT signalling pathway. Oncol Rep 39: 2402-2412, 2018

25. Wang Z, Yao YJ, Zheng F, Guan Z, Zhang L, Dong N and Qin WJ: Mir-138-5p acts as a tumor suppressor by targeting pyruvate dehydrogenase kinase 1 in human retinoblastoma. Eur Rev Med Pharmacol Sci 21: 5624-5629, 2017.

26. Yang L, Wei N, Wang L, Wang X and Liu QH: miR-498 promotes cell proliferation and inhibits cell apoptosis in retinoblastoma by directly targeting CCPG1. Childs Nerv Syst 34: 417-422, 2018.

27. Zhao Y, Zhang S and Zhang Y: MicroRNA-320 inhibits cell proliferation, migration and invasion in retinoblastoma by targeting specificity protein 1. Mol Med Rep 16: 2191-2198, 2017

28. Mao Y, Chen H, Lin Y, Xu X, Hu Z, Zhu Y, Wu J, Xu X, Zheng X and Xie L: microRNA-330 inhibits cell motility by downregulating Sp1 in prostate cancer cells. Oncol Rep 30: 327-333, 2013.

29. Zheng Z, Bao F, Chen $X$, Huang $H$ and Zhang $X$ : MicroRNA-330-3p expression indicates good prognosis and suppresses cell proliferation by targeting Bmi-1 in osteosarcoma. Cell Physiol Biochem 46: 442-450, 2018.

30. Xu W, Jiang H, Zhang F, Gao J and Hou J: MicroRNA-330 inhibited cell proliferation and enhanced chemosensitivity to 5 -fluorouracil in colorectal cancer by directly targeting thymidylate synthase. Oncol Lett 13: 3387-3394, 2017.

31. Zhang C, Zhang S, Zhang Z, He J, Xu Y and Liu S: ROCK has a crucial role in regulating prostate tumor growth through interaction with c-Myc. Oncogene 33: 5582-5591, 2014.

32. Rossman KL, Der CJ and Sondek J: GEF means go: Turning on RHO GTPases with guanine nucleotide-exchange factors. Nat Rev Mol Cell Biol 6: 167-180, 2005.

33. Patel RA, Forinash KD, Pireddu R, Sun Y, Sun N, Martin MP, Schönbrunn E, Lawrence NJ and Sebti SM: RKI-1447 is a potent inhibitor of the Rho-associated ROCK kinases with anti-invasive and antitumor activities in breast cancer. Cancer Res 72: 5025-5034, 2012.

34. Whatcott CJ, Ng S, Barrett MT, Hostetter G, Von Hoff DD and Han H: Inhibition of ROCK1 kinase modulates both tumor cells and stromal fibroblasts in pancreatic cancer. PLoS One 12: e0183871, 2017.

35. Wang X, Huang Y, Guo R, Liu Y, Qian Y, Liu D, Dai X, Wei Z, Jin F and Liu Y: Clinicopathological significance of ROCK1 and PIK3CA expression in nasopharyngeal carcinoma. Exp Ther Med 13: 1064-1068, 2017.

36. Wu D, Niu X, Pan H, Zhou Y, Qu P and Zhou J: MicroRNA-335 is downregulated in bladder cancer and inhibits cell growth, migration and invasion via targeting ROCK1. Mol Med Rep 13: 4379-4385, 2016

37. Zhang S, Zhao Y and Wang L: MicroRNA-198 inhibited tumorous behaviors of human osteosarcoma through directly targeting ROCK1. Biochem Biophys Res Commun 472: 557-565, 2016. 